\title{
MIXING PROPERTIES OF A CLASS OF BERNOULLI-PROCESSES
}

\author{
DORIS FIEBIG
}

\begin{abstract}
We prove that stationary very weak Bernoulli processes with rate $O(1 / n)($ VWB $O(1 / n))$ are strictly very weak Bernoulli with rate $O(1 / n)$. Furthermore we discuss the relation between VWB $O(1 / n)$ and the classical mixing properties for countable state processes. In particular, we show that VWB $O(1 / n)$ implies $\phi$-mixing.
\end{abstract}

\section{INTRODUCTION}

Let $X_{i}:(\Omega, \mathscr{A}, \mu) \rightarrow(S, \mathscr{B}), i \in \mathbf{Z}$, be a stationary sequence of random variables on a probability space $(\Omega, \mathscr{A}, \mu)$ with values in a Polish space $(S, \mathscr{B})$. In this setting we define very weak Bernoulli processes with rate $\varepsilon(n)$, denoted by $\operatorname{VWB} \varepsilon(n)$, and strictly $\operatorname{VWB} \varepsilon(n)$ processes. It was shown by Dehling, Denker and Philipp [D.D.P] that $O(1 / n)$ is the fastest rate for which nonindependent VWB-processes exist. We show that $\left(X_{i}\right)_{i \in \mathbf{Z}}$ is $\operatorname{VWB} O(1 / n)$ iff $\left(X_{i}\right)_{i \in \mathbf{Z}}$ is strictly VWB $O(1 / n)$. This strengthens the result of Eberlein, that real-valued strictly VWB $O(1 / n)$ processes with certain moment conditions satisfy an almost sure invariance principle [E]. Then we restrict ourselves to the discrete case, i.e., we assume $S$ to be countable and that $\mathscr{B}$ is generated by the discrete metric. Our main result in this case is that $\operatorname{VWB} O(1 / n)$ implies $\phi$ mixing, which improves an earlier result of [D.D.P]. We show that VWB $O(1 / n)$ gives no constraints on the $\phi$-mixing rate, and that $\mathrm{VWB} O(1 / n)$ does not imply $\psi$-mixing. After that we give a new upper bound for the Wasserstein-distance, which implies that a $\phi$-mixing process with $\phi$-mixing rate $\phi(i)$ is strictly VWB with rate $\frac{1}{n} \sum_{i=1}^{n} \phi(i)$; in particular $\phi$-mixing processes with summable rates are VWB $O(1 / n)$.

\section{VWB $O(1 / n)$ IMPLIES STRICTLY VWB $O(1 / n)$}

Let $X_{i}:(\Omega, \mathscr{A}, \mu) \rightarrow(S, \mathscr{B}), i \in \mathbf{Z}$, be a stationary sequence of random variables. Let $\sigma: S \times S \rightarrow \mathbf{R}$ be a metric, such that $\mathscr{B}$ is generated by $\sigma$ and $S$ is a Polish space. For $-\infty \leq m \leq n \leq \infty$ let $\mathscr{A}_{m}^{n}=\mathscr{A}\left(X_{i}, m \leq i \leq n\right)$ be the $\sigma$-algebra generated by $X_{i}$ with indices between $m$ and $n$. For two probability measures $\nu_{1}, \nu_{2}$ on $\left(S^{n}, B^{n}\right)$ let $P_{n}\left(\nu_{1}, \nu_{2}\right)=\left\{\lambda: B^{n} \times B^{n} \rightarrow[0,1]: \lambda\right.$ is a probability measure with $i$ th marginal $\left.\nu_{i}, i=1,2\right\}$. So $P_{n}\left(\nu_{1}, \nu_{2}\right)$ is the set of joinings of $\nu_{1}$ and $\nu_{2}$. Then, for $Z \in \mathscr{A}_{-\infty}^{0}$ with $\mu(Z)>0$, define the

Received by the editors February 5, 1990 and, in revised form, April 13, 1991.

1980 Mathematics Subject Classification (1985 Revision). Primary 60G10; Secondary 60F99. 
Wasserstein-distance

$$
\rho_{n}(\mu, \mu(\cdot / Z)):=\inf \int_{S^{n} \times S^{n}} \frac{1}{n} \sum_{i=1}^{n} \sigma\left(x_{i}, y_{i}\right) d \lambda\left(x_{1}, \ldots, x_{n}, y_{1}, \ldots, y_{n}\right)
$$

where the infimum is taken over $\lambda \in P_{n}\left(\left(X_{1}, \ldots, X_{n}\right) \mu,\left(X_{1}, \ldots, X_{n}\right) \mu(\cdot / Z)\right)$.

Definition 1 [E]. $\left(X_{i}\right)_{i \in Z}$ is very weak Bernoulli with rate $\varepsilon(n) \quad(\mathrm{VWB} \varepsilon(n))$ iff

(1) $\varepsilon(n) \rightarrow 0, n \rightarrow \infty$,

(2) $\forall n \in \mathbf{N} \quad \forall m \in \mathbf{Z}^{+} \quad \exists D=D(m, n) \in \mathscr{A}_{-m}^{0}$ with

$$
\begin{gathered}
\mu(D) \geq 1-\varepsilon(n), \\
A \subset D, A \in \mathscr{A}_{-m}^{0}, \quad \mu(A)>0 \Rightarrow \rho_{n}(\mu, \mu(\cdot / A)) \leq \varepsilon(n) .
\end{gathered}
$$

Definition 2 [E]. $\left(X_{i}\right)_{i \in \mathbf{Z}}$ is strictly $\operatorname{VWB} \varepsilon(n)$ iff $\left(X_{i}\right)_{i \in \mathbf{Z}}$ is $\mathrm{VWB} \varepsilon(n)$ and all sets $D(m, n)$ can be chosen to be $\Omega$, i.e., $\rho_{n}(\mu, \mu(\cdot / A)) \leq \varepsilon(n) \forall A \in \mathscr{A}_{-\infty}^{0}$.

We shall tacitly assume $\mu(A)>0$ when dealing with conditional probabilities as $\mu(\cdot / A)$.

In [D.D.P] it was shown that a $\operatorname{VWB} \varepsilon(n)$ process with $\lim \inf n \varepsilon(n)=0$ is already independent. This means that $\varepsilon(n)=O(1 / n)$ is the fastest rate for which one can possibly have a nonindependent $\operatorname{VWB} \varepsilon(n)$ process. Various classes of examples for $\operatorname{VWB} O(1 / n)$ processes are given in $[\mathrm{F}]$. They include $m$-dependent processes, finite state mixing Markov chains and continuous factors of finite state mixing Markov chains. There it was shown that the VWB $O(1 / n)$-property is not preserved under finitary factor maps, not even if the coding length of the factor map has moments of all orders $[F]$.

Our main interest is the examination of VWB $O(1 / n)$ processes. The fundamental observation is the following:

Theorem 3 [F]. Let $\left(X_{i}\right)_{i \in \mathbf{Z}}$ be a stationary sequence of random variables with values in a Polish space. Let $0 \leq M<\infty$. Then:

$\left(X_{i}\right)_{i \in \mathbf{Z}}$ is $V W B$ with rate $M / n$ iff $\left(X_{i}\right)_{i \in \mathbf{Z}}$ is strictly $V W B$ with rate $M / n$.

We need

Lemma 4 [S]. Let $M_{n}=\left\{\nu:\left(S^{n}, B^{n}\right) \rightarrow R: \nu\right.$ probability measure $\}$ with weak topology. Then

$$
\begin{aligned}
n \rho_{n}: M_{n} \times M_{n} & \rightarrow \mathbf{R} \\
\left(\nu_{1}, \nu_{2}\right) & \rightarrow n \rho_{n}\left(\nu_{1}, \nu_{2}\right)
\end{aligned}
$$

is a lower semicontinuous function.

Proof of Theorem 3. Let $0 \leq M<\infty$. Let $\left(X_{i}\right)_{i \in \mathbf{Z}}$ be VWB with rate $M / n$. Then for all $n \in \mathbf{N}, m \in \overline{\mathbf{Z}}^{+}$sets $D(m, n) \in \mathscr{A}_{-m}^{0}$ can be chosen such that (1.1), (1.2) hold for $\varepsilon(n)=M / n$. We show that the process is strictly VWB $M / n$. Let $n \in \mathbf{N}, m \in \mathbf{Z}^{+}$and choose a set $A \in \mathscr{A}_{-m}^{0}$ with $\mu(A)>0$. Now pick $k_{0} \geq n$ such that $\mu(A \cap D(m, k))>\mu(A) / 2>0 \quad \forall k \geq k_{0}$. Then $\mu(\cdot / A \cap D(m, k)) \rightarrow \mu(\cdot / A)$ in weak topology. Because $A \cap D(m, k) \subset D(m, k)$ and $A \cap D(m, k) \in \mathscr{A}_{-m}^{0} \forall k,(1.2)$ implies

$$
\rho_{k}(\mu, \mu(\cdot / A \cap D(m, k))) \leq \frac{M}{k} \quad \forall k \geq k_{0} .
$$


Since $n \leq k_{0}$ we have

$$
\rho_{n}(\mu, \mu(\cdot / A \cap D(m, k))) \leq \frac{M}{n} \quad \forall k \geq k_{0} .
$$

By Lemma 4 this implies $\rho_{n}(\mu, \mu(\cdot / A)) \leq M / n$, so the process is strictly VWB $M / n$. The converse is trivial.

Theorem 3 does not hold for rates slower than $O(1 / n)$. This was shown in $[\mathrm{F}]$ and we recall here the example:

Let $\left(X_{i}\right)_{i \in \mathbf{Z}}$ be a Markov chain with state space $\mathbf{Z}^{+}$and transition probabilities

$$
p_{i j}= \begin{cases}1, & i=j+1, j \geq 0 \\ c_{j}, & i=0, j \geq 0 \\ 0, & \text { otherwise }\end{cases}
$$

where $\left(c_{n}\right)_{n \geq 0}$ is a sequence with $c_{j} \geq 0 \forall j, c_{j}>0$ infinitely often, $\sum_{j=1}^{\infty} j c_{j}<$ $\infty$. Then the $\left(p_{i j}\right)_{i, j \in \mathbf{Z}^{+}}$define a stationary Markov chain. For stationary Markov chains one can calculate the exact value of $\rho_{n}\left(\mu, \mu\left(\cdot / X_{0}=i\right)\right)$ for all $n \in \mathbf{N}, i \in \mathbf{Z}^{+}$(by Theorem 6). This gives the possibility by choosing $\left(c_{j}\right)_{j \geq 0}$ to achieve a given VWB rate $\varepsilon(n)$ with $n \varepsilon(n) \rightarrow \infty$. In [F] it was shown that the Markov chain above is not strictly VWB, i.e., there is no rate $\varepsilon(n)$ for which $\left(X_{i}\right)_{i \in \mathbf{Z}}$ is strictly $\mathrm{VWB} \varepsilon(n)$.

\section{Relating VWB $O(1 / n)$ to the Classical MiXiNg PROPERTies}

Now we want to examine the mixing properties of $\operatorname{VWB} O(1 / n)$ processes. Because $\varepsilon(n)=O(1 / n)$ is the fastest rate for which one can have nonindependent $\mathrm{VWB} \varepsilon(n)$ processes, and because of Theorem 3, one expects that these processes have good mixing properties, but this depends strongly on the state space $S$ and the metric $\sigma$. There exists a stationary $\operatorname{VWB} O(1 / n)$ process with uncountable state space $S \subset \mathbf{R}$, where $\sigma$ is the Euclidean metric, which is not even $\alpha$-mixing [B1], but on the other hand finite state $\operatorname{VWB} O(1 / n)$ processes are always weak Bernoulli [D.D.P]. From now on we restrict ourselves to stationary processes with at most countable state space, endowed with the discrete metric. For $Z \in \mathscr{A}_{-\infty}^{0}, \mu(Z)>0$ and $1 \leq i \leq n<\infty$ we define the distribution distance of names by

$$
\begin{aligned}
& \left|\operatorname{dist} X_{i}^{n} \mu-\operatorname{dist} X_{i}^{n} \mu(\cdot / Z)\right| \\
& \quad:=\frac{1}{2} \sum_{\left(y_{i}, \ldots, y_{n}\right) \in S^{n-i+1}}\left|\mu\left(X_{n}=y_{n}, \ldots, X_{i}=y_{i}\right)-\mu\left(X_{n}=y_{n}, \ldots, X_{i}=y_{i} / Z\right)\right| .
\end{aligned}
$$

With this notation we have the simple, but extremely useful

Lemma 5. Let $\left(n_{i}\right)_{i \in \mathbf{N}}$ be a strictly increasing sequence of natural numbers with $n_{0}:=0$. Let $Z \in \mathscr{A}_{-\infty}^{0}$ with $\mu(Z)>0$. Then for all $N \in \mathbf{N}$

$$
n_{N+1} \rho_{n_{N+1}}(\mu, \mu(\cdot / Z)) \geq \sum_{i=0}^{N}\left|\operatorname{dist} X_{n_{i}+1}^{n_{i+1}} \mu-\operatorname{dist} X_{n_{i}+1}^{n_{i+1}} \mu(\cdot / Z)\right| \text {. }
$$

(If $S$ is countable and the metric $\sigma$ is bounded below by $\varepsilon$, i.e., $\sigma(x, y) \geq$ $\varepsilon>0 \quad \forall x \neq y$, then this lemma holds with the RHS multiplied by $\varepsilon)$. The next theorem gives a new upper bound for the Wasserstein-distance. 
Theorem 6. Let $n \in \mathbf{N}, Z \in \mathscr{A}_{-\infty}^{0}$ with $\mu(Z)>0$. Then

$$
n \rho_{n}(\mu, \mu(\cdot / Z)) \leq \sum_{i=1}^{n}\left|\operatorname{dist} X_{i}^{n} \mu-\operatorname{dist} X_{i}^{n} \mu(\cdot / Z)\right| \text {. }
$$

The proof of Theorem 6 is deferred to the Appendix. It depends on the construction of a joining $\nu_{n}$ of $\left(X_{1}, \ldots, X_{n}\right) \mu$ and $\left(X_{1}, \ldots, X_{n}\right) \mu(\cdot / Z)$ such that

$$
\begin{gathered}
\int_{S^{n} \times S^{n}} \sigma\left(x_{i}, y_{i}\right) d \nu_{n}\left(\left(x_{1}, \ldots, x_{n}\right),\left(y_{1}, \ldots, y_{n}\right)\right) \\
\leq\left|\operatorname{dist} X_{i}^{n} \mu-\operatorname{dist} X_{i}^{n} \mu(\cdot / Z)\right| \quad \forall 1 \leq i \leq n .
\end{gathered}
$$

The joining $\nu_{n}$ is a generalisation of a construction in [F], and shows that for Markov chains

$$
n \rho_{n}\left(\mu, \mu\left(\cdot / X_{0}=x\right)\right)=\sum_{i=1}^{n}\left|\operatorname{dist} X_{i} \mu-\operatorname{dist} X_{i} \mu\left(\cdot / X_{0}=x\right)\right| \text {. }
$$

We use the following mixing coefficients:

$$
\begin{aligned}
& \alpha(n):=\sup _{A \in \mathscr{A}_{-\infty}^{0}} \sup _{B \in \mathscr{P}_{n}^{\infty}}|\mu(B \cap A)-\mu(B) \mu(A)|, \\
& \mathrm{WB}(n):=\sup _{m, k \geq 0} \sum_{B \in \mathscr{P}_{n}^{n+k}} \sum_{A \in \mathscr{P}_{-m}^{0}} \mu(A) \cdot|\mu(B \mid A)-\mu(B)|,
\end{aligned}
$$

where $\mathscr{P}_{n}^{n+k}$ (resp. $\mathscr{P}_{-m}^{0}$ ) is the finest partition of $\Omega$ into sets $B \in \mathscr{A}_{n}^{n+k}$ (resp. $A \in \mathscr{A}_{-m}^{0}$ ).

$$
\begin{aligned}
& \phi(n):=\sup _{A \in \mathscr{\mathscr { A }}_{-\infty}^{0}} \sup _{B \in \mathscr{A}_{n}^{\infty}}|\mu(B / A)-\mu(B)|, \\
& \psi(n):=\sup _{A \in \mathscr{A}_{-\infty}^{0}} \sup _{B \in \mathscr{A}_{n}^{\infty}}|\mu(B / A) / \mu(B)-1|
\end{aligned}
$$

(where as always $\mu(A)>0$ is assumed, if necessary). $\left(X_{i}\right)_{i \in \mathbf{Z}}$ is said to be $\alpha$-mixing (weak Bernoulli (=WB), $\phi$-mixing or $\psi$-mixing) iff $\alpha(n) \rightarrow 0$ $(\mathrm{WB}(n) \rightarrow 0, \phi(n) \rightarrow 0, \psi(n) \rightarrow 0)$, respectively. From the definitions of the mixing coefficients it is clear that

$$
\psi \text {-mixing } \Rightarrow \phi \text {-mixing } \Rightarrow \mathrm{WB} \Rightarrow \alpha \text {-mixing. }
$$

The reverse implications do not hold. For general background on the properties of these mixing coefficients see [B3]. We first strengthen the result of [D.D.P] to

Theorem 7. Let $\left(X_{i}\right)_{i \in \mathbf{Z}}$ be a stationary process with at most countable state space $S$ and discrete metric (or a metric bounded away from zero). Then $\left(X_{i}\right)_{i \in \mathbf{Z}} \mathrm{VWB} O(1 / n) \Rightarrow\left(X_{i}\right)_{i \in \mathbf{Z}} \phi$-mixing.

For the proof we need the following Lemma 8 , which is an easy consequence of the observation that VWB with rate $M / n$ implies $(m \in N)$

$$
\begin{aligned}
M & \geq(N m) \rho_{N m}(\mu, \mu(\cdot / D)) \\
& \geq \sum_{i=1}^{N}\left|\operatorname{dist} X_{(i-1) m+1}^{i m} \mu-\operatorname{dist} X_{(i-1) m+1}^{i m} \mu(\cdot / D)\right| \quad \text { by Lemma } 5 .
\end{aligned}
$$


So that, given $\varepsilon>0$, there is an $N \in \mathbf{N}$ such that for any set

$$
\begin{aligned}
& D \in \mathscr{A}_{-\infty}^{0}, \mu(D)>0 \text { there is an } i \leq N \text { with } \\
& \left|\operatorname{dist} X_{(i-1) m+1}^{i m} \mu-\operatorname{dist} X_{(i-1) m+1}^{i m} \mu(\cdot / D)\right|<\varepsilon .
\end{aligned}
$$

Lemma 8. Let $\left(X_{i}\right)_{i \in \mathrm{Z}}$ be $V W B$ with rate $\varepsilon(n)=M / n, 0 \leq M<\infty$. Let $\sigma: S^{\mathbf{Z}} \rightarrow S^{\mathbf{Z}}$ be the shift map, i.e., $\sigma\left(\left(s_{j}\right)_{j \in \mathbf{Z})_{i}=s_{i+1}} \forall i \in \mathbf{Z}\right.$. Fix $r \in \mathbf{N}$, $m \in \mathbf{N}$. Choose $A_{1}, \ldots, A_{r} \in \mathscr{A}_{1}^{m}$ with $\mu\left(A_{s}\right)>0 \forall s$. Fix $\delta>0$. Then there is $k=k\left(\min _{1 \leq s \leq r} \mu\left(A_{s}\right), \delta\right) \in \mathbf{N}$ such that:

$$
\begin{aligned}
& \left.\forall D \in \mathscr{A}_{-\infty}^{0}, \mu(D)>0 \exists 0 \leq i \leq k \text { (i depends on } D\right) \text { with } \\
& \left|\mu\left(\sigma^{-i m} A_{s} / D\right)-\mu\left(A_{s}\right)\right|<\delta \mu\left(A_{s}\right) \quad \forall s \leq r .
\end{aligned}
$$

Proof. Choose $\varepsilon<\delta \min _{1 \leq s \leq r} \mu\left(A_{s}\right)$, and apply the observation (2.2) above, using the fact that $\left|\mu\left(\sigma^{-i m} A_{s} / D\right)-\mu\left(A_{s}\right)\right| \leq\left|\operatorname{dist} X_{i m+1}^{(i+1) m} \mu-\operatorname{dist} X_{i m+1}^{(i+1) m} \mu(\cdot / D)\right|$.

Remark. Lemma 8 remains valid for strictly $\mathrm{VWB} \varepsilon(n)$ processes, for all rates $\varepsilon(n)$.

Proof of Theorem 7. Let $\left(X_{i}\right)_{i \in \mathbf{Z}}$ be VWB $\varepsilon(n), \varepsilon(n)=M / n, M<\infty$. Assume $\left(X_{i}\right)_{i \in \mathbf{Z}}$ is not $\phi$-mixing.

Claim 1. $\forall m \in \mathbf{N} \quad \forall \varepsilon>0 \quad \exists 1=l_{0}<l_{1}<l_{2}<\cdots<l_{m}<\infty \quad \exists k \in \mathbf{N}$ $\exists B_{i} \in \mathscr{A}_{l_{i-1}}^{l_{i}-1}, 1 \leq i \leq m$ and $\exists C \in \mathscr{A}_{-k}^{0}, \mu(C)>0$, such that $\mu\left(B_{i}\right)>1-\varepsilon$, $\mu\left(B_{i} / C\right)<\varepsilon \quad \forall i \in\{1, \ldots, m\}$.

We prove this claim by induction on $m$. For $m=1$, we apply Theorem 1 of [B2], so $\left(X_{i}\right)_{i \in \mathbf{Z}}$ not $\phi$-mixing means $\phi(1)=1$. This implies the claim for $m=1$, because one can approximate sets in $\mathscr{A}_{1}^{\infty}$ (resp. $\mathscr{A}_{-\infty}^{0}$ ) arbitrarily well by sets in $\mathscr{A}_{1}^{l}$ (resp. $\mathscr{A}_{-k}^{0}$ ) for $l$ (resp. $k$ ) large enough.

Let $\varepsilon>0$ and pick $0<\delta<\varepsilon / 3$.

By hypothesis there are $1=l_{0}<l_{1}<\cdots<l_{m}<\infty$ and sets $B_{i} \in \mathscr{A}_{l_{i-1}}^{l_{i}-1}$, $1 \leq i \leq m, C \in \mathscr{A}_{-s}^{0}, \mu(C)>0$ with $\mu\left(B_{i}\right)>1-\delta, \mu\left(B_{i} / C\right)<\delta$. We shall show that there are sets $B_{m+1}$ and $E$ and that $B_{1}, \ldots, B_{m}, B_{m+1}$ and $E$ satisfy the claim for $m+1$ and $\varepsilon$. Let $I:=\left\{i \in\{1, \ldots, m\}: \mu\left(B_{i} / C\right)>\right.$ $0\}, A_{i}:=\sigma^{-s-1}\left(B_{i} \cap C\right), 1 \leq i \leq m$. Then $A_{i} \in \mathscr{A}_{1}^{l_{m}+s} \forall i$. We apply Lemma 8 to the $\left\{A_{i}, i \in I\right\} \cup\left\{\sigma^{-s-1} C\right\}$ with $m^{\prime}:=l_{m}+s$. So we get $k=k\left(\mu(C),\left(\mu\left(A_{i}\right)\right)_{i \in I} ; \frac{1}{2}\right)$ such that for any set $D \in \mathscr{A}_{-\infty}^{0} \exists 0 \leq j \leq k$ such that

$$
\begin{aligned}
& \left|\mu\left(\sigma^{-j m^{\prime}} A_{i} / D\right)-\mu\left(A_{i}\right)\right|<\frac{1}{2} \mu\left(A_{i}\right) \quad \forall i \in I, \\
& \left|\mu\left(\sigma^{-j m^{\prime}-s-1} C / D\right)-\mu(C)\right|<\frac{1}{2} \mu(C) .
\end{aligned}
$$

Now, because not $\phi$-mixing means in particular $\phi(n)=1 \forall n$, we find for $0<\delta_{1}<\delta$ with $2 \delta_{1} / \mu(C)<\delta$ a number $L \geq(2 k+1) m^{\prime}+2$ and sets

$$
B \in \mathscr{A}_{(2 k+1) m^{\prime}}^{L}, \quad D \in \mathscr{A}_{-L}^{0} \quad \text { with } \mu(B)>1-\delta_{1}, \quad \mu(B / D)<\delta_{1} .
$$

Let $E:=C \cap \sigma^{j m^{\prime}+s+1} D$ where $j$ is according to (2.3). Then $E \in \mathscr{A}_{-L-j m^{\prime}-s-1}^{0}$, and $\mu(E)=\mu(D) \mu\left(\sigma^{-j m^{\prime}-s-1} C / D\right)>\frac{1}{2} \mu(D) \mu(C)>0$ by (2.3). Let $B_{m+1}:=$ $\sigma^{j m^{\prime}+s+1} B$, so $B_{m+1} \in \mathscr{A}_{(2 k-j) m^{\prime}}^{L-j m^{\prime}-s-1}$ and $(2 k-j) m^{\prime} \geq k m^{\prime} \geq l_{m}$, so for $l_{m+1}:=$ $L-j m^{\prime}-s-1$ we have

$$
B_{m+1} \in \mathscr{A}_{l_{m}}^{l_{m+1}} \text { and } \mu\left(B_{m+1}\right)>1-\delta .
$$


For $i \in\{1, \ldots, m\}-I$ we have

$$
\mu\left(B_{i} / E\right) \leq \frac{\mu\left(B_{i} \cap C\right)}{\mu(E)}=0<\delta .
$$

For $i \in I$ we have

$$
\begin{aligned}
\mu\left(B_{i} / E\right) & =\frac{\mu\left(B_{i} \cap C \cap \sigma^{j m^{\prime}+s+1} D\right)}{\mu(E)}=\frac{\mu(D) \mu\left(\sigma^{-j m^{\prime}} A_{i} / D\right)}{\mu(E)} \\
& =\frac{\mu\left(\sigma^{-j m^{\prime}} A_{i} / D\right)}{\mu\left(\sigma^{-j m^{\prime}-s-1} C / D\right)} \\
& \left.\leq \frac{\frac{3}{2} \mu\left(A_{i}\right)}{\frac{1}{2} \mu(C)}=3 \mu\left(B_{i} / C\right)<3 \delta \quad \text { (because of }(2.3)\right)
\end{aligned}
$$

and

$$
\begin{aligned}
\mu\left(B_{m+1} / E\right) & =\frac{\mu\left(\sigma^{j m^{\prime}+s+1} B \cap C \cap \sigma^{j m^{\prime}+s+1} D\right)}{\mu\left(C \cap \sigma^{j m^{\prime}+s+1} D\right)} \leq \frac{\mu(B / D) \mu(D)}{\mu\left(C \cap \sigma^{j m^{\prime}+s+1} D\right)} \\
& \left.=\frac{\mu(B / D)}{\mu\left(\sigma^{-j m^{\prime}-s-1} C / D\right)}<\frac{\delta_{1}}{\frac{1}{2} \mu(D)}<\delta \quad \text { (because of }(2.3),(2.4)\right) .
\end{aligned}
$$

Because $3 \delta<\varepsilon$ we have sets $B_{1}, \ldots, B_{m+1}$ and $E$ which satisfy Claim 1 for $m+1$ and $\varepsilon$. This proves Claim 1 .

Now we choose $\varepsilon<\frac{1}{2}$ and $m \in \mathbf{N}$ such that $m(1-2 \varepsilon)>M$. Then we choose sets $B_{i}, C$ from Claim 1 to obtain by Lemma 5 the estimate

$$
\begin{aligned}
M & \geq l_{m} \rho_{l_{m}}(\mu, \mu(\cdot / C)) \geq \sum_{i=1}^{m}\left|\operatorname{dist} X_{l_{i-1}}^{l_{i}-1} \mu-\operatorname{dist}\right| X_{l_{i-1}}^{l_{i}-1} \mu(\cdot / C) \mid \\
& \geq \sum_{i=1}^{m}\left|\mu\left(B_{i}\right)-\mu\left(B_{i} / C\right)\right| \geq m(1-2 \varepsilon)>M .
\end{aligned}
$$

This contradiction shows, $\left(X_{i}\right)_{i \in \mathbf{Z}}$ was, in fact, $\phi$-mixing and proves the theorem.

Remark. The key to the proof of Theorem 7 is Claim 1. In fact, one can prove Claim 1 for all strictly $\mathrm{VWB} \varepsilon(n)$ processes, but of course, the fastest rate $\varepsilon(n)=O(1 / n)$ was needed to produce a contradiction from Claim 1 . We show in $\S 3$ that for each sequence $\varepsilon(n), i n \varepsilon(n) \rightarrow \infty, \varepsilon(n) \rightarrow 0$ there is a strictly $\mathrm{VWB} \varepsilon(n)$ process which is not $\phi$-mixing.

Theorem 7 is the strongest possible, since there exists $\operatorname{VWB} O(1 / n)$, a finite state process, which is not $\phi$-mixing (see $[F])$.

Example 9. There exist a VWB $O(1 / n)$ process with countable state space which is not $\psi$-mixing. Let $0<p<1$ and for $i, j \in \mathbf{Z}^{+}$let

$$
p_{i j}:= \begin{cases}p, & \text { if } j=i+1, i \geq 0 \\ 1-p, & \text { if } i \geq 0, j=0 \\ 0, & \text { otherwise. }\end{cases}
$$

This stochastic matrix defines a stationary Markov chain $\left(X_{i}\right)_{i \in \mathbf{Z}}$ with state space $\mathbf{Z}^{+}$and invariant measure $\mu$, where $\mu\left(X_{0}=i\right)=(1-p) \cdot p^{i}, i \geq 0$.

$\left(X_{i}\right)_{i \in \mathbf{Z}}$ is not $\psi$-mixing, because $\mu\left(X_{n}=n+1 / X_{0}=0\right)=0 \quad \forall n$. 
$\left(X_{i}\right)_{i \in \mathbf{Z}}$ is $\phi$-mixing, as an easy calculation shows, so $\left(X_{i}\right)_{i \in \mathbf{Z}}$ is $\operatorname{VWB} O(1 / n)$, see Corollary 13.

The next theorem shows that $\operatorname{VWB} O(1 / n)$ has no constraints on the $\phi$ mixing rate.

Theorem 10. Let $\left(\lambda_{n}\right)_{n \geq 1}$ be a sequence with $\lambda_{1} \leq 1,\left(\lambda_{n}\right)_{n \geq 1}$ nonincreasing, $\lambda_{n} \rightarrow 0$ as $n \rightarrow \infty$ and $-\log \left(1-\lambda_{n}\right)$ is convex on the set $\left\{k: \lambda_{k}<1\right\}$. Then there exists a countable state process $\left(X_{i}\right)_{i \in \mathrm{Z}}$ which is $\operatorname{VWB} O(1 / n)$ and $\phi$-mixing with $\frac{1}{2} \lambda_{n} \leq \phi(n) \leq \lambda_{n}$.

Proof. Kesten and O'Brien have constructed an example in [K.O'B] (which we discuss in $\S 3)$, where one easily checks that $\lambda_{n}=\mu\left(\bigcup_{k \geq n}\left\{U_{k} \geq k\right\}\right)$. So $\lambda_{n} \rightarrow 0$ means $E U_{0}<\infty$ in their construction. Apply Theorems 14 and 15 .

We do not expect the converse of Theorem 7 to be true, but we do have the following corollary from Theorem 6 .

Corollary 11. Let $\left(X_{i}\right)_{i \in \mathbf{Z}}$ be $\phi$-mixing with $\phi$-mixing rate $\phi(n)$, then $\left(X_{i}\right)_{i \in \mathbf{Z}}$ is strictly $\mathrm{VWB} \varepsilon(n)$ for $\varepsilon(n)=\frac{1}{n} \sum_{i=1}^{n} \phi(i)$.

Proof. Theorem 6 yields

$$
\begin{aligned}
\sup _{Z \in \mathscr{A}_{-\infty}^{0}} n \rho_{n}(\mu, \mu(\cdot / Z)) & \leq \sup _{Z \in \mathscr{A}_{-\infty}^{0}} \sum_{i=1}^{n}\left|\operatorname{dist} X_{i}^{n} \mu-\operatorname{dist} X_{i}^{n} \mu(\cdot / Z)\right| \\
& \leq \sup _{Z \in \mathscr{A}_{-\infty}^{0}} \frac{1}{2} \sum_{i=1}^{n}\left(\left|\mu\left(B_{i}^{+}\right)-\mu\left(B_{i}^{+} / Z\right)\right|+\left|\mu\left(B_{i}^{-}\right)-\mu\left(B_{i}^{-} / Z\right)\right|\right) \\
& \leq \sum_{i=1}^{n} \phi(i)
\end{aligned}
$$

where

$$
B_{i}^{+}:=\left\{\left(y_{i}, \ldots, y_{n}\right): \mu\left(X_{i}=y_{i}, \ldots, X_{n}=y_{n}\right) \geq \mu\left(X_{i}=y_{i}, \ldots, X_{n}=y_{n} / Z\right)\right\}
$$

and

$$
\begin{aligned}
B_{i}^{-}:=\left\{\left(y_{i}, \ldots, y_{n}\right): \mu\left(X_{i}=y_{i},\right.\right. & \left., X_{n}=y_{n}\right) \\
& \left.<\mu\left(X_{i}=y_{i}, \ldots, X_{n}=y_{n} / Z\right)\right\} .
\end{aligned}
$$

In particular, we have the following consequences.

Corollary 12. If $\left(X_{i}\right)_{i \in \mathbf{Z}}$ is $\phi$-mixing with $\sum_{i=1}^{\infty} \phi(i)<\infty$, then $\left(X_{i}\right)_{i \in \mathbf{Z}}$ is VWB $O(1 / n)$.

Corollary 13. Let $\left(X_{i}\right)_{i \in \mathbf{Z}}$ be a stationary Markov chain with at most countable state space. Then $\left(X_{i}\right)_{i \in \mathrm{Z}}$ is $\mathrm{VWB} O(1 / n)$ iff $\left(X_{i}\right)_{i \in \mathrm{Z}}$ is $\phi$-mixing.

Proof. If $\left(X_{i}\right)_{i \in \mathbf{Z}}$ is $\phi$-mixing then $\phi(n)=O\left(\lambda^{n}\right)$ for a $0<\lambda<1$ [R]. Apply Corollary 12 .

\section{SOME ASPECTS OF STRICTLY VWB $\varepsilon(n)$ PROCESSES}

We want to discuss a class of examples, which was given by Kesten and O'Brien in its original form. These examples will show that $\varepsilon(n)=O(1 / n)$ is the only VWB rate which forces the process to be $\phi$-mixing. 
We use the notation of [K.O'B].

Let $\left(U_{i}\right)_{i \in \mathbf{Z}}$ be i.i.d. with values in $\mathbf{Z}^{+}$.

Let $\left(V_{i}\right)_{i \in \mathbf{Z}}$ be i.i.d. with values in $\{0,1\}, \mu\left(V_{0}=0\right)=\frac{1}{2}$.

Let $\left(U_{i}\right)_{i \in \mathbf{Z}}$ be independent from $\left(V_{i}\right)_{i \in \mathbf{Z}}$.

The process which Kesten and O'Brien constructed is $X_{n}:=\left(U_{n}, V_{n}, V_{n-U_{n}}\right)$, $n \in \mathbf{Z}$. In this section $\left(X_{i}\right)_{i \in \mathbf{Z}}$ is always this process.

Kesten and O'Brien proved

Theorem 14 [K.O'B]. If $\phi(n)$ is the $\phi$-mixing coefficient for $\left(X_{i}\right)_{i \in \mathbf{Z}}$ then

$$
\frac{1}{2} \mu\left(\bigcup_{k \geq n}\left\{U_{k} \geq k\right\}\right) \leq \phi(n) \leq \mu\left(\bigcup_{k \geq n}\left\{U_{k} \geq k\right\}\right) .
$$

In particular $\left(X_{i}\right)_{i \in \mathbf{Z}}$ is $\phi$-mixing $\Leftrightarrow E U_{0}<\infty$.

We prove an analogous estimate for the VWB-rate.

Theorem 15. $\left(X_{i}\right)_{i \in \mathrm{Z}}$ is strictly $\operatorname{VWB} \varepsilon(n)$ where

$$
\frac{1}{2 n} \sum_{k=1}^{n} \mu\left(U_{k} \geq k\right) \leq \varepsilon(n) \leq \frac{1}{n} \sum_{k=1}^{n} \mu\left(U_{k} \geq k\right) .
$$

Proof. First we observe that

$$
n \rho_{n}(\mu, \mu(\cdot / Z)) \geq \sum_{i=1}^{n}\left|\operatorname{dist} X_{i} \mu-\operatorname{dist} X_{i} \mu(\cdot / Z)\right|
$$

by Lemma 5 . Thus

$$
\begin{aligned}
& n \varepsilon(n) \geq \sup _{Z \in \mathscr{A}_{-\infty}^{0}} \sum_{i=1}^{n}\left|\operatorname{dist} X_{i} \mu-\operatorname{dist} X_{i} \mu(\cdot / Z)\right| \\
& \geq \sup _{Z \in \mathscr{A}_{-\infty}^{0}} \sum_{i=1}^{n}\left|\mu\left(U_{i} \geq i, V_{i-U_{i}}=1\right)-\mu\left(U_{i} \geq i, V_{i-U_{i}}=1 / Z\right)\right| \\
& \geq \sum_{i=1}^{n}-\frac{1}{2} \mu\left(U_{i} \geq i\right) \quad\left(Z_{N}=\bigcap_{j=0}^{N}\left\{V_{-j}=0\right\} \text {, let } N \rightarrow \infty\right) \text {. }
\end{aligned}
$$

So

$$
\varepsilon(n) \geq \frac{1}{2 n} \sum_{i=1}^{n} \mu\left(U_{i} \geq i\right) .
$$

For proving the upper bound we cannot apply Theorem 6, because for large $n$ we have for $\mu\left(U_{k}=k\right):=1 / k(k+1), k \geq 1$,

$$
\sup _{Z \in \mathscr{P}_{-\infty}^{0}} \sum_{i=1}^{n}\left|\operatorname{dist} X_{i}^{n} \mu-\operatorname{dist} X_{i}^{n} \mu(\cdot / Z)\right| \geq \frac{1}{8} \sum_{k=1}^{n} \mu\left(\bigcup_{i \geq k} U_{i} \geq i\right)=\frac{1}{8} n .
$$

Because $\frac{1}{n} \sum_{i=1}^{n} \mu\left(U_{i} \geq i\right) \rightarrow 0$ if $n \rightarrow \infty$, we have for large $n$

$$
\frac{1}{n} \sum_{i=1}^{n} \mu\left(U_{i} \geq i\right) \leq \frac{1}{8} \leq \frac{1}{n} \sup _{Z \in \mathscr{Q}_{-\infty}^{0}} \sum_{i=1}^{n}\left|\operatorname{dist} X_{i}^{n} \mu-\operatorname{dist} X_{i}^{n} \mu(\cdot / Z)\right| .
$$


Thus Theorem 6 is not strong enough in this case, because it gives a trivial upper bound. So we have to construct a measure

$$
\lambda \in P_{n}\left(\left(X_{1}, \ldots, X_{n}\right) \mu(\cdot / Z),\left(X_{1}, \ldots, X_{n}\right) \mu\right) .
$$

Fix $n, m \in \mathbf{Z}^{+}$and $Z \in \mathscr{A}_{-m}^{0}$ of the form $Z=\left\{U_{0}=u_{0}, V_{0}=v_{0}, V_{0-U_{0}}=\right.$ $\left.w_{0}, \ldots, U_{-m}=u_{-m}, V_{-m}=v_{-m}, V_{-m-U_{-m}}=w_{-m}\right\}$ such that $\mu(Z)>0$. First we have, because $\left(U_{i}\right)_{i \in \mathbf{Z}},\left(V_{i}\right)_{i \in \mathbf{Z}}$ are i.i.d.,

$$
n \rho_{n}\left(\left(U_{1}, \ldots, U_{n}, V_{1}, \ldots, V_{n}\right) \mu,\left(U_{1}, \ldots, U_{n}, V_{1}, \ldots, V_{n}\right) \mu(\cdot / Z)\right)=0 .
$$

If $U_{k}<k$ or $\left(U_{k}>k+m\right.$ and $\left.k-U_{k} \neq-i-U_{-i} \forall 0 \leq i \leq m\right)$ then $V_{k-U_{k}}$ is independent of $Z \in \mathscr{A}_{-m}^{0}$, and it is this property which helps us to find a good joining.

Let $C=\left\{U_{1}=u_{1}, V_{1}=v_{1}, \ldots, U_{n}=u_{n}, V_{n}=v_{n}\right\} .(3.1)$ implies $\mu(C)=$ $\mu(C / Z)$.

Let $J(C)$ be the indices where $C$ does not hit $Z$, so $J(C):=\{1 \leq l \leq n$ : $u_{1}<l$ or $\left(u_{l}>l+m\right.$ and $\left.\left.l-u_{l} \neq-i-u_{-i} \forall 0 \leq i \leq m\right)\right\}$. Then $J(C)=\varnothing$ or $J(C)=\left\{j_{1}, \ldots, j_{r}\right\}$ and $\left(X_{j_{1}}, \ldots, X_{j_{r}}\right) \mu(\cdot / C)=\left(X_{j_{1}}, \ldots, X_{j_{r}}\right) \mu(\cdot / C \cap Z)$.

If $J(C)=\{1, \ldots, n\}$ then there is $\lambda_{C}:\{0,1\}^{n} \times\{0,1\}^{n} \rightarrow \mathbf{R}$ such that

(1) $\operatorname{pr}_{1} \lambda_{C}\left(\left(x_{1}, \ldots, x_{n}\right)\right)=\mu\left(V_{1-U_{1}}=x_{1}, \ldots, V_{n-U_{n}}=x_{n} / C \cap Z\right)$,

(2) $\operatorname{pr}_{2} \lambda_{C}\left(\left(y_{1}, \ldots, y_{n}\right)\right)=\mu\left(V_{1-U_{1}}=y_{1}, \ldots, V_{n-U_{n}}=y_{n} / C\right)$ and

$$
\int \sum_{i=1}^{n} \sigma\left(x_{i}, y_{i}\right) d \lambda_{C}=0
$$
let

If $J(C) \neq\{1, \ldots, n\}$ then $\{1, \ldots, n\}-J(C)=\left\{l_{1}, \ldots, l_{s}\right\}, s \geq 1$. Then

$$
\overline{w_{i}}:= \begin{cases}v_{l_{i}-u_{l_{i}}}, & \text { if } l_{i}-u_{l_{i}} \geq-m \\ w_{-r}, & \text { if } l_{i}-u_{l_{i}}=-r-u_{-r} \text { for } r \in\{0, \ldots, m\} .\end{cases}
$$

So we have $\left(\bar{w}_{1}, \ldots, \bar{w}_{s}\right) \in\{0,1\}^{s}$. Let $\lambda_{C}:\{0,1\}^{n} \times\{0,1\}^{n} \rightarrow \mathbf{R}$ be defined by

$$
\begin{aligned}
\lambda_{C}\left(\left(x_{1}, \ldots, x_{n}\right),\left(y_{1}, \ldots, y_{n}\right)\right):= & 0 \text { if }\left(x_{l_{1}}, \ldots, x_{l_{s}}\right) \neq\left(\bar{w}_{1}, \ldots, \bar{w}_{s}\right) \text { or } \\
& y_{i} \neq x_{i} \text { for some } i \in J(C), \\
\lambda_{C}\left(\left(x_{1}, \ldots, x_{n}\right),\left(y_{1}, \ldots, y_{n}\right)\right):= & \mu\left(V_{1-U_{1}}=x_{1}, \ldots, V_{n-U_{n}}=x_{n} / C \cap Z\right) \\
& \cdot \mu\left(V_{l_{1}-U_{l_{1}}}=y_{l_{1}}, \ldots, V_{l_{s}-U_{l_{s}}} / C\right) \text { otherwise. }
\end{aligned}
$$

Then one calculates

$$
\begin{aligned}
& \operatorname{pr}_{1} \lambda_{C}\left(\left(x_{1}, \ldots, x_{n}\right)\right)=\mu\left(V_{1-U_{1}}=x_{1}, \ldots, V_{n-U_{n}}=x_{n} / C \cap Z\right), \\
& \operatorname{pr}_{2} \lambda_{C}\left(\left(y_{1}, \ldots, y_{n}\right)\right)=\mu\left(V_{1-U_{1}}=y_{1}, \ldots, V_{n-U_{n}}=y_{n} / C\right), \text { and }
\end{aligned}
$$




$$
\begin{aligned}
& \int \sum_{i=1}^{n} \sigma\left(x_{i}, y_{i}\right) d \lambda_{C} \\
&=\sum_{\left\{\left(x_{1}, \ldots, x_{n}\right): x_{l_{i}}=\bar{w}_{i} i \leq s\right\}} \sum_{\left\{\left(y_{1}, \ldots, y_{n}\right): y_{i}=x_{i} \text { if } i \in J(C)\right\}} \sum_{i=1}^{n} \sigma\left(x_{i}, y_{i}\right) \\
& \cdot \mu\left(V_{1-U_{1}}=x_{1}, \ldots, V_{n-U_{n}}=x_{n} / C \cap Z\right) \\
& \cdot \mu\left(V_{l_{1}-U_{l_{1}}}=y_{l_{1}}, \ldots, V_{l_{s}-U_{l_{s}}}=y_{l_{s}} / C\right) \\
&= \sum_{\left\{\left(x_{1}, \ldots, x_{n}\right): x_{l_{i}}=\bar{w}_{i} i \leq s\right\}} \sum_{\left\{\left(y_{1}, \ldots, y_{n}\right): y_{i}=x_{i} \text { if } i \in J(C)\right\}} \sum_{r=1}^{s} \sigma\left(x_{l_{r}}, y_{l_{r}}\right) \\
& \cdot \mu\left(V_{1-U_{1}}=x_{1}, \ldots, V_{n-U_{n}}=x_{n} / C \cap Z\right) \\
& \cdot \mu\left(V_{l_{1}-U_{l_{1}}}=y_{l_{1}}, \ldots, V_{l_{s}-U_{l_{s}}}=y_{l_{s}} / C\right) \\
& \leq \sum_{\left\{\left(x_{1}, \ldots, x_{n}\right): x_{l_{i}}=\bar{w}_{i} i \leq s\right\}} s \mu\left(V_{1-U_{1}}=x_{1}, \ldots, V_{n-U_{n}}=x_{n} / C \cap Z\right) \\
& \leq s=\operatorname{card} J(C)^{C} .
\end{aligned}
$$

So we get with $\lambda:\left(\mathbf{Z}^{+} \times\{0,1\} \times\{0,1\}\right)^{n} \times\left(\mathbf{Z}^{+} \times\{0,1\} \times\{0,1\}\right)^{n} \rightarrow \mathbf{R}$ defined by

$$
\begin{aligned}
& \lambda\left(\left(\left(u_{1}, v_{1}, w_{1}\right), \ldots,\left(u_{n}, v_{n}, w_{n}\right)\right) \times\left(\left(a_{1}, b_{1}, c_{1}\right), \ldots,\left(a_{n}, b_{n}, c_{n}\right)\right)\right) \\
& \quad:=\mu(C) \cdot \lambda_{C}\left(\left(w_{1}, \ldots, w_{n}\right),\left(c_{1}, \ldots, c_{n}\right)\right),
\end{aligned}
$$

$C$ as above, if $u_{i}=a_{i}, v_{i}=b_{i} \forall i$ and

$$
\lambda\left(\left(\left(u_{1}, v_{1}, w_{1}\right), \ldots,\left(u_{n}, v_{n}, w_{n}\right)\right) \times\left(\left(a_{1}, b_{1}, c_{1}\right), \ldots,\left(a_{n}, b_{n}, c_{n}\right)\right)\right):=0
$$

otherwise, a probability measure $\lambda \in P_{n}\left(\left(X_{1}, \ldots, X_{n}\right) \mu(\cdot / Z),\left(X_{1}, \ldots, X_{n}\right) \mu\right)$ by $(3.1)$ and

$$
\begin{aligned}
n \rho_{n}(\mu, \mu(\cdot / Z)) & \leq \int \sum_{i=1}^{n} \sigma\left(x_{i}, y_{i}\right) d \lambda=\sum_{C} \mu(C) \int \sum_{i=1}^{n} \sigma\left(x_{i}, y_{i}\right) d \lambda_{C} \\
& \leq \sum_{C} \mu(C) \cdot \operatorname{card} J(C)^{c} \leq \sum_{C} \mu(C) \cdot \operatorname{card}\left(\left\{i \leq n: U_{i} \geq i\right\} \cap C\right) \\
& =\sum_{i=1}^{n} \mu\left(U_{i} \geq i\right) .
\end{aligned}
$$

Remark. One can actually strengthen this last construction and prove that if $\left(U_{i}\right)_{i \in \mathbf{Z}}$ is a stationary process with values in $\mathbf{Z}^{+}$and $\left(V_{i}\right)_{i \in \mathbf{Z}}$ is a stationary process with values in $\{0,1\}$ and $X_{n}:=\left(U_{n}, V_{n}, V_{n-U_{n}}\right)$ then

(1) $\left(X_{i}\right)_{i \in \mathbf{Z}} \phi$-mixing $\Leftrightarrow E U_{0}<\infty,\left(U_{i}\right)_{i \in \mathbf{Z}} \phi$-mixing, $\left(V_{i}\right)_{i \in \mathbf{Z}} \phi$-mixing,

(2) $\left(X_{i}\right)_{i \in \mathbf{Z}} \operatorname{VWB} O(1 / n) \Leftrightarrow E U_{0}<\infty,\left(U_{i}\right)_{i \in \mathbf{Z}} \operatorname{VWB} O(1 / n)$, $\left(V_{i}\right)_{i \in \mathbf{Z}} \operatorname{VWB} O(1 / n)$.

For this one needs a Borel-Cantelli-Lemma for $\phi$-mixing sequences.

We get as corollaries of Theorems 14 and 15: 
Corollary 16. $X_{n}:=\left(U_{n}, V_{n}, V_{n-U_{n}}\right)$ as above. Then

$$
\left(X_{i}\right)_{i \in \mathbf{Z}} \phi \text {-mixing } \Leftrightarrow\left(X_{i}\right)_{i \in \mathbf{Z}} \operatorname{VWB} O(1 / n) \Leftrightarrow E U_{0}<\infty .
$$

Corollary 17. For any rate $\varepsilon(n)$ with $(n+1) \varepsilon(n+1)-n \varepsilon(n) \leq n \varepsilon(n)-$ $(n-1) \varepsilon(n-1) \quad \forall n, \varepsilon(n) \rightarrow 0, n \varepsilon(n) \rightarrow \infty$ and $n \varepsilon(n) \leq n \quad \forall n$ there is a process $\left(X_{i}\right)_{i \in \mathbf{Z}}$ which is strictly $\mathrm{VWB} \varepsilon(n)$ and not $\phi$-mixing.

We would like to find an example of a process which is not VWB $O(1 / n)$, but $\phi$-mixing, but we have not yet been successful. We believe a good candidate is the following:

Let $\left(U_{i}\right)_{i \in \mathbf{Z}},\left(V_{i}\right)_{i \in \mathbf{Z}}$ as above. Let $Y_{n}:=\left(V_{n}, V_{n-U_{n}}\right), n \in \mathbf{Z}$. Then it is not hard to see that $E U_{0}=\infty \Rightarrow\left(Y_{i}\right)_{i \in Z}$ is not $\operatorname{VWB} O(1 / n)$. The conjecture is

$$
E U_{0}=\infty, \quad \sum_{k=1}^{\infty} \mu\left(U_{k} \geq k\right)^{2}<\infty \Rightarrow\left(Y_{i}\right)_{i \in \mathbf{Z}} \text { is } \phi \text {-mixing. }
$$

\section{APPENDIX}

Proof of Theorem 6. Fix $n \in \mathbf{N}, Z \in \mathscr{A}_{-\infty}^{0}, \mu(Z)<0$. We will need some elaborate notation. Let $\left\{X_{1}^{n}=s_{1}^{n}\right\}:=\left\{X_{1}=s_{1}, \ldots, X_{n}=s_{n}\right\}$.

$$
\begin{aligned}
& I_{1}:=\left\{\left(s_{1}, \ldots, s_{n}\right) \in S^{n}: \mu\left(X_{1}^{n}=s_{1}^{n}\right)>\mu\left(X_{1}^{n}=s^{n} / Z\right)\right\}, \\
& \bar{I}_{1}:=\left\{\left(s_{1}, \ldots, s_{n}\right) \in S^{n}: \mu\left(X_{1}^{n}=s_{1}^{n}\right)<\mu\left(X_{1}^{n}=s_{1}^{n} / Z\right)\right\}, \\
& \tau_{1}\left(s_{1}, \ldots, s_{n}\right):=\mu\left(X_{1}^{n}=s_{1}^{n}\right), \quad \bar{\tau}_{1}\left(s_{1}, \ldots, s_{n}\right):=\mu\left(X_{1}^{n}=s_{1}^{n} / Z\right), \\
& \rho_{1}\left(s_{1}, \ldots, s_{n}\right):=\left(\mu\left(X_{1}^{n}=s_{1}^{n}\right)-\mu\left(X_{1}^{n}=s_{1}^{n} / Z\right)\right) \cdot 1_{I_{1}}\left(s_{1}, \ldots, s_{n}\right), \\
& \bar{\rho}_{1}\left(s_{1}, \ldots, s_{n}\right):=\left(\mu\left(X_{1}^{n}=s_{1}^{n} / Z\right)-\mu\left(X_{1}^{n}=s_{1}^{n}\right)\right) \cdot 1_{\bar{I}_{1}}\left(s_{1}, \ldots, s_{n}\right) .
\end{aligned}
$$

Then inductively for $1 \leq k \leq n-1$

$$
\begin{aligned}
& \tau_{k+1}\left(s_{k+1}, \ldots, s_{n}\right):=\sum_{\left(s_{1}, \ldots, s_{k}\right)} \rho_{k}\left(s_{1}, \ldots, s_{k}, s_{k+1}, \ldots, s_{n}\right), \\
& \bar{\tau}_{k+1}\left(s_{k+1}, \ldots, s_{n}\right):=\sum_{\left(s_{1}, \ldots, s_{k}\right)} \bar{\rho}_{k}\left(s_{1}, \ldots, s_{k}, s_{k+1}, \ldots, s_{n}\right), \\
& I_{k+1}=\left\{\left(s_{k+1}, \ldots, s_{n}\right) \in S^{n-k}: \tau_{k+1}\left(s_{k+1}, \ldots, s_{n}\right)>\bar{\tau}_{k+1}\left(s_{k+1}, \ldots, s_{n}\right)\right\}, \\
& \bar{I}_{k+1}=\left\{\left(s_{k+1}, \ldots, s_{n}\right) \in S^{n-k}: \tau_{k+1}\left(s_{k+1}, \ldots, s_{n}\right)<\bar{\tau}_{k+1}\left(s_{k+1}, \ldots, s_{n}\right)\right\}, \\
& \rho_{k+1}\left(s_{1}, \ldots, s_{n}\right):=\rho_{k}\left(s_{1}, \ldots, s_{n}\right)\left(1-\frac{\bar{\tau}_{k+1}\left(s_{k+1}, \ldots, s_{n}\right)}{\tau_{k+1}\left(s_{k+1}, \ldots, s_{n}\right)}\right) 1_{I_{k+1}}\left(s_{k+1}, \ldots, s_{n}\right), \\
& \bar{\rho}_{k+1}\left(s_{1}, \ldots, s_{n}\right):=\bar{\rho}_{k}\left(s_{1}, \ldots, s_{n}\right)\left(1-\frac{\tau_{k+1}\left(s_{k+1}, \ldots, s_{n}\right)}{\bar{\tau}_{k+1}\left(s_{k+1}, \ldots, s_{n}\right)}\right) 1_{\bar{I}_{k+1}}\left(s_{k+1}, \ldots, s_{n}\right), \\
& \tau_{n+1}:=\sum_{s \in S^{n}} \rho_{n}(s), \quad \bar{\tau}_{n+1}:=\sum_{s \in S^{n}} \bar{\rho}_{n}(s) .
\end{aligned}
$$

We want to define a probability measure on $S^{n} \times S^{n}$, therefore we partition the 
set $S^{n} \times S^{n}=W_{0} \cup W_{1} \cup \cdots \cup W_{n} \cup R$ in disjoint sets, where

$$
\begin{aligned}
& W_{0}=\left\{(x, x): x \in S^{n}\right\}, \\
& W_{n}=\left\{\left(\left(x_{1}, \ldots, x_{n}\right),\left(y_{1}, \ldots, y_{n}\right)\right): x_{n} \neq y_{n}, \rho_{n}\left(x_{1}, \ldots, x_{n}\right)>0\right. \text { and } \\
& \left.\bar{\rho}_{n}\left(y_{1}, \ldots, y_{n}\right)>0\right\}, \\
& W_{i}=\left\{\left(\left(x_{1}, \ldots, x_{n}\right), \begin{array}{r}
\left.\left(y_{1}, \ldots, y_{n}\right)\right): x_{i} \neq y_{i}, x_{r}=y_{r}, i<r \leq n, \\
\left.\min \left(\tau_{i+1}\left(x_{i+1}, \ldots, x_{n}\right), \bar{\tau}_{i+1}\left(x_{i+1}, \ldots, x_{n}\right)\right)>0\right\} \\
\text { for } 1 \leq i \leq n-1,
\end{array}\right.\right. \\
& R:=S^{n} \times S^{n}-\bigcup_{i=0}^{n} W_{i} .
\end{aligned}
$$

Then we define $\nu_{n}: S^{n} \times S^{n} \rightarrow \mathbf{R}$ in the following way:

(1) $\left(\left(s_{1}, \ldots, s_{n}\right),\left(s_{1}, \ldots, s_{n}\right)\right) \in W_{0}$ :

$\nu_{n}\left(\left(s_{1}, \ldots, s_{n}\right),\left(s_{1}, \ldots, s_{n}\right)\right):=\min \left(\tau_{1}\left(s_{1}, \ldots, s_{n}\right), \bar{\tau}_{1}\left(s_{1}, \ldots, s_{n}\right)\right)$.

(2) $\left(\left(a_{1}, \ldots, a_{i}, s_{i+1}, \ldots, s_{n}\right),\left(b_{1}, \ldots, b_{i}, s_{i+1}, \ldots, s_{n}\right)\right) \in W_{i}, 1 \leq i$ $\leq n-1$ :

$$
\begin{aligned}
& \nu_{n}\left(\left(a_{1}, \ldots, a_{i}, s_{i+1}, \ldots, s_{n}\right),\left(b_{1}, \ldots, b_{i}, s_{i+1}, \ldots, s_{n}\right)\right) \\
&:= \frac{\min \left(\tau_{i+1}\left(s_{i+1}, \ldots, s_{n}\right), \bar{\tau}_{i+1}\left(s_{i+1}, \ldots, s_{n}\right)\right)}{\tau_{i+1}\left(s_{i+1}, \ldots, s_{n}\right) \bar{\tau}_{i+1}\left(s_{i+1}, \ldots, s_{n}\right)} \\
& \cdot \rho_{i}\left(a_{1}, \ldots, a_{i}, s_{i+1}, \ldots, s_{n}\right) \bar{\rho}_{i}\left(b_{1}, \ldots, b_{i}, s_{i+1}, \ldots, s_{n}\right) .
\end{aligned}
$$

(3) $\left(\left(a_{1}, \ldots, a_{n}\right),\left(b_{1}, \ldots, b_{n}\right)\right) \in W_{n}$ :

$$
\begin{aligned}
& \nu_{n}\left(\left(a_{1}, \ldots, a_{n}\right),\left(b_{1}, \ldots, b_{n}\right)\right) \\
& \quad:=\rho_{n}\left(a_{1}, \ldots, a_{n}\right) \bar{\rho}_{n}\left(b_{1}, \ldots, b_{n}\right) \frac{\min \left(\tau_{n+1}, \bar{\tau}_{n+1}\right)}{\tau_{n+1} \bar{\tau}_{n+1}} .
\end{aligned}
$$

(4) $\nu_{n}\left(\left(a_{1}, \ldots, a_{n}\right),\left(b_{1}, \ldots, b_{n}\right)\right):=0$ if $\left(\left(a_{1}, \ldots, a_{n}\right),\left(b_{1}, \ldots, b_{n}\right)\right) \in$ $R$. We use the abbreviated notation $s^{(i)}:=\left(s_{i}, \ldots, s_{n}\right) \in S^{n-i+1}, 1 \leq i \leq n$. First we want to prove that $\nu_{n}$ is a joining of

$$
\left(X_{1}, \ldots, X_{n}\right) \mu \text { and }\left(X_{1}, \ldots, S_{n}\right) \mu(\cdot / Z) \text {. }
$$

One calculates

$$
\begin{aligned}
\alpha\left(s^{(1)}\right):= & \sum_{t^{(1)} \in S^{n}} \nu_{n}\left(s^{(1)}, t^{(1)}\right) \\
= & \nu_{n}\left(s^{(1)}, s^{(1)}\right)+\sum_{i=1}^{n-1}\left\{\sum_{\left\{t^{(1)} \in S^{n}: t_{i} \neq s_{i}, t_{i+1}=s_{i+1}, \ldots, t_{n}=s_{n}\right\}} \nu_{n}\left(s^{(1)}, t^{(1)}\right)\right. \\
& +\sum_{\left\{t^{(1)} \in S^{n}: t_{n} \neq s_{n}\right\}} \nu_{n}\left(s^{(1)}, t^{(1)}\right) \\
= & \min \left(\tau_{1}\left(s^{(1)}\right), \bar{\tau}_{1}\left(s^{(1)}\right)\right)+\sum_{i=1}^{n-1} \frac{\min \left(\tau_{i+1}\left(s^{(i+1)}\right), \bar{\tau}_{i+1}\left(s^{(i+1)}\right)\right)}{\tau_{i+1}\left(s^{(i+1)}\right)} \rho_{i}\left(s^{(1)}\right) \\
& +\frac{\min \left(\tau_{n+1}, \bar{\tau}_{n+1}\right)}{\tau_{n+1}} \rho_{n}\left(s^{(1)}\right) .
\end{aligned}
$$

To calculate $\alpha\left(s^{(1)}\right)$ we have to look for the set $I_{k}$ that $s^{(k)}$ belongs to: 
Case 1. $s^{(1)} \notin I_{1}$. Then $\rho_{i}\left(s^{(1)}\right)=0 \quad \forall i \geq 1$, so

$$
\alpha\left(s^{(1)}\right)=\tau_{1}\left(s^{(1)}\right)=\mu\left(X_{1}=s_{1}, \ldots, X_{n}=s_{n}\right) .
$$

Case 2. $s^{(1)} \in I_{1}, s^{(2)} \notin I_{2}$. Then $\rho_{i}\left(s^{(1)}\right)=0 \quad \forall i \geq 2$, and

$$
\alpha\left(s^{(1)}\right)=\bar{\tau}_{1}\left(s^{(1)}\right)+\rho_{1}\left(s^{(1)}\right)=\mu\left(X_{1}=s_{1}, \ldots, X_{n}=s_{n}\right) .
$$

General case. $s^{(1)} \in I_{1}, \ldots, s^{(k)} \in I_{k}, s^{(k+1)} \notin I_{k+1}$. Then the same argument as in Case 2 shows $\alpha\left(s^{(1)}\right)=\mu\left(X_{1}=s_{1}, \ldots, X_{n}=s_{n}\right)$ and in the case $s^{(1)} \in I_{1}, \ldots, s^{(n)} \in I_{n}$ one uses the fact $\tau_{n+1}=\bar{\tau}_{n+1}$ to see $\alpha\left(s^{(1)}\right)$ $=\mu\left(X_{1}=s_{1}, \ldots, X_{n}=s_{n}\right)$. Similarly

$$
\sum_{s^{(1)} \in S^{n}} \nu_{n}\left(s^{(1)}, t^{(1)}\right)=\mu\left(X_{1}=t_{1}, \ldots, X_{n}=t_{n} / Z\right) .
$$

For proving (2.1) we need an equivalent definition of the sets $I_{k}$.

Claim 2. $1 \leq k \leq n$. Then

$$
I_{k}=\left\{s^{(k)}: \mu\left(X_{k}=s_{k}, \ldots, X_{n}=s_{n}\right)>\mu\left(X_{k}=s_{k}, \ldots, X_{n}=s_{n} / Z\right)\right\} .
$$

Proof of the claim.

$$
\begin{aligned}
\tau_{k}\left(s^{(k)}\right) & =\sum_{s_{1}, \ldots, s_{k-1}} \rho_{k-1}\left(s_{1}, \ldots, s_{k-1}, s_{k}, \ldots, s_{n}\right) \\
& =\sum_{s_{1}, \ldots, s_{k-2}} \sum_{s_{k-1}} \rho_{k-2}\left(s^{(1)}\right)\left(1-\frac{\bar{\tau}_{k-1}\left(s^{(k-1)}\right)}{\tau_{k-1}\left(s^{(k-1)}\right)}\right) 1_{I_{k-1}}\left(s^{(k-1)}\right) \\
& =\sum_{\left\{s:\left(s, s_{k}, \ldots, s_{n}\right) \in I_{k-1}\right\}}\left(\tau_{k-1}\left(s, s_{k}, \ldots, s_{n}\right)-\bar{\tau}_{k-1}\left(s, s_{k}, \ldots, s_{n}\right)\right) \\
& =\sum_{\left\{s:\left(s, s^{(k)}\right) \in I_{k-1}\right\}}\left(\tau_{k-1}\left(s, s^{(k)}\right)-\bar{\tau}_{k-1}\left(s, s^{(k)}\right)\right) .
\end{aligned}
$$

So we get

$$
\begin{aligned}
\tau_{k}\left(s^{(k)}\right)> & \bar{\tau}_{k}\left(s^{(k)}\right) \\
\Leftrightarrow & \sum_{\left\{s:\left(s, s^{(k)}\right) \in I_{k-1}\right\}}\left(\tau_{k-1}\left(s, s^{(k)}\right)-\bar{\tau}_{k-1}\left(s, s^{(k)}\right)\right) \\
& >\sum_{\left\{s:\left(s, s^{(k)}\right) \in \bar{I}_{k-1}\right\}}\left(\bar{\tau}_{k-1}\left(s, s^{(k)}\right)-\tau_{k-1}\left(s, s^{(k)}\right)\right) \\
\Leftrightarrow & \sum_{s \in S} \tau_{k-1}\left(s, s^{(k)}\right)>\sum_{s \in S} \bar{\tau}_{k-1}\left(s, s^{(k)}\right) \\
\Leftrightarrow & \sum_{s_{1}, \ldots, s_{k-1}} \tau_{1}\left(s_{1}, \ldots, s_{k-1}, s^{(k)}\right)>\sum_{s_{1}, \ldots, s_{k-1}} \bar{\tau}_{1}\left(s_{1}, \ldots, s_{k-1}, s^{(k)}\right)
\end{aligned}
$$

by repeating the argument

$$
\Leftrightarrow \mu\left(X_{k}=s_{k}, \ldots, X_{n}=s_{n}\right)>\mu\left(X_{k}=s_{k}, \ldots, X_{n}=s_{n} / Z\right)
$$

This completes the proof of the claim. 
Now we compute for $1 \leq i \leq n$

$$
\begin{aligned}
& \int_{S^{n} \times S^{n}} \sigma\left(x_{i}, y_{i}\right) d \nu_{n}\left(\left(x_{1}, \ldots, x_{n}\right),\left(y_{1}, \ldots, y_{n}\right)\right) \\
& =\nu_{n}\left(\left\{\left(s^{(1)}, t^{(1)}\right): s_{i} \neq t_{i}\right\}\right) \\
& \leq 1-\sum_{j=0}^{i-1} \nu_{n}\left(\left\{\left(s^{(1)}, t^{(1)}\right): s_{j+1}=t_{j+1}, \ldots, s_{n}=t_{n}, s_{j} \neq t_{j}\right\}\right) \\
& =1-\sum_{j=0}^{i-1} \sum_{s^{(j+1)}} \min \left(\tau_{j+1}\left(s^{(j+1)}\right), \bar{\tau}_{j+1}\left(s^{(j+1)}\right)\right) \\
& =1-\sum_{j=1}^{i-1}\left(\sum_{s^{(j)} \in I_{j}} \bar{\tau}_{j}\left(s^{(j)}\right)+\sum_{s^{(j)} \notin I_{j}} \tau_{j}\left(s^{(j)}\right)\right) \\
& -\left(\sum_{s^{(i)} \in I_{i}} \sum_{s_{1}}, \ldots, s_{i-1} \bar{\rho}_{i-1}\left(s^{(1)}\right)+\sum_{s^{(i)} \notin I_{i}} \sum_{s_{1}, \ldots, s_{i-1}} \rho_{i-1}\left(s^{(1)}\right)\right) \\
& =1-\sum_{j=1}^{i-2}\left(\sum_{s^{(j)} \in I_{j}} \bar{\tau}_{j}\left(s^{(j)}\right)+\sum_{s^{(j)} \notin I_{j}} \tau_{j}\left(s^{(j)}\right)\right) \\
& -\sum_{s^{(i-1)} \in I_{i-1}} \bar{\tau}_{i-1}\left(s^{(i-1)}\right)-\sum_{s^{(i-1)} \notin I_{i-1}} \tau_{i-1}\left(s^{(i-1)}\right) \\
& -\sum_{s^{(i)} \in I_{i}, s^{(i-1)} \in \bar{I}_{i-1}}\left(\bar{\tau}_{i-1}\left(s^{(i-1)}\right)-\tau_{i-1}\left(s^{(i-1)}\right)\right) \\
& -\sum_{s^{(i)} \notin I_{i}, s^{(i-1)} \in I_{i-1}}\left(\tau_{i-1}\left(s^{(i-1)}\right)-\bar{\tau}_{i-1}\left(s^{(i-1)}\right)\right) \\
& =1-\sum_{j=1}^{i-2}\left(\sum_{s^{(j)} \in I_{j}} \bar{\tau}_{j}\left(s^{(j)}\right)+\sum_{s^{(j)} \notin I_{j}} \tau_{j}\left(s^{(j)}\right)\right) \\
& -\sum_{\left\{s^{(i-1)}: s^{(i)} \in I_{i}\right\}} \bar{\tau}_{i-1}\left(s^{(i-1)}\right)-\sum_{\left\{s^{(i-1)}: s^{(i)} \notin I_{i}\right\}} \tau_{i-1}\left(s^{(i-1)}\right) \\
& =1-\sum_{\left\{s^{(1)}: s^{(i)} \in I_{i}\right\}} \bar{\tau}_{1}\left(s^{(1)}\right)-\sum_{\left\{s^{(1)}: s^{(i)} \notin I_{i}\right\}} \tau_{1}\left(s^{(1)}\right) \quad \text { (by repeating the argument) } \\
& =1-\sum_{s^{(i)} \in I_{i}} \mu\left(X_{i}=s_{i}, \ldots, X_{n}=s_{n} / Z\right)-\sum_{s^{(i)} \notin I_{i}} \mu\left(X_{i}=s_{i}, \ldots, X_{n}=s_{n}\right) \\
& =\sum_{s^{(i)} \in I_{i}}\left(\mu\left(X_{i}=s_{i}, \ldots, X_{n}=s_{n}\right)-\mu\left(X_{i}=s_{i}, \ldots, X_{n}=s_{n} / Z\right)\right) \\
& =\left|\operatorname{dist} X_{i}^{n} \mu-\operatorname{dist} X_{i}^{n} \mu(\cdot / Z)\right| \quad \text { (by Claim 2). }
\end{aligned}
$$

So (2.1) is proved and therefore Theorem 6, also. 


\section{REFERENCES}

[B1] R. C. Bradley, On a very weak Bernoulli condition, Stochastic 13 (1984), 61-81.

[B2] - On the $\phi$-mixing condition for stationary random sequences, Duke Math. J. 47 (1980), 421-433.

[B3] _ Basic properties of strong mixing conditions: Dependence in Probability and Statistics (E. Eberlein and M. S. Taqqu, eds.), Birkhäuser, 1986, pp. 165-192.

[D.D.P] H. Dehling, M. Denker, and W. Philipp, Versik processes and very weak Bernoulli processes with summable rates are independent, Proc. Amer. Math. Soc. 91 (1984), 618-623.

[E] E. Eberlein, Strong approximation of very weak Bernoulli processes, Z. Wahrsch. Verw. Gebiete 62 (1983), 17-37.

[F] D. Fiebig, Über very weak Bernoulli Prozesse mit schnellen Raten, Dissertation, Göttingen, 1988.

[K.O'B] H. Kesten and G. L. O'Brien, Examples of mixing sequences, Duke Math. J. 43 (1976), 405-415.

[R] M. Rosenblatt, Markov processes, structure and asymptotic behavior, Springer-Verlag, Berlin, 1971.

[S.] W. Strittmatter, Measures of dependence for processes in metric spaces, Stochastics Stochastics Rep. 27 (1989), 33-50.

Universität Heidelberg SFB 123, Im Neuenheimer Feld 294, D-6900 Heidelberg 1, West GERMANY

Current address: Institut für Angewandte Mathematik, Universität Heidelberg, Im Neuenheimer Feld 294, 6900 Heidelberg, Germany

E-mail address: bq6@dhdurzl.bitnet 